\title{
The effects of complex decongestive therapy on kinesthetic sense of hands, upper extremity function, and quality of life in patients with breast cancer-related lymphedema
}

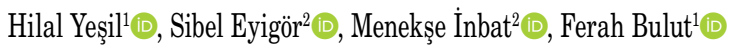 \\ ${ }^{1}$ Department of Physical Medicine and Rehabilitation, Afyon Kocatepe University, Faculty of Medicine, Afyonkarahisar, Turkey \\ ${ }^{2}$ Department of Physical Medicine and Rehabilitation, Ege University, Faculty of Medicine, Izmir, Turkey
}

Received: August 29, 2019 Accepted: March 13, 2020 Published online: May 25, 2021

\begin{abstract}
Objectives: This study aims to investigate the effect of complex decongestive therapy (CDT) on the kinesthetic sense of hands, upper extremity function, and the quality of life in patients with breast cancer-related lymphedema (BCRL).

Patients and methods: Between August 2018 and August 2019, total of 50 women with BCRL (mean age: $56.5 \pm 9.6$ years; range, 36 to 71 years) were included in the study. Kinesthetic sense of the hand, upper extremity function (Disabilities of the Arm, Shoulder and Hand [DASH]), quality of life (European Organization for Research and Treatment of Cancer Quality of Life Core Questionnaire [EORTC QLQ-C30]), and arm volume of all patients were evaluated before and after the treatment. All patients received CDT for 20 sessions for $1 \mathrm{~h}$ over a total of four weeks.

Results: A statistically significant decrease in the volume of the involved extremity was observed after the treatment $(\mathrm{p}<0.001)$. There was a significant decrease in the symptom score $(\mathrm{p}<0.001)$ and a significant improvement in the general health status and functional scales of the EORTC QLQ-C30 ( $<<0.001$ and $\mathrm{p}=0.012$, respectively). The DASH scores and visual and kinesthetic sense scores of the patients significantly improved after the treatment $(\mathrm{p}=0.016, \mathrm{p}=0.008$, and $\mathrm{p}<0.001$, respectively).

Conclusion: Our study results show that BCRL is a serious complication which may lead to impairment in the kinesthetic sense of hand and upper extremity function with the increased arm volume. The CDT is an effective and safe method not only to achieve significant volume reduction in the extremities, but also to achieve favorable results in managing these problems.
\end{abstract}

Keywords: Complex decongestive therapy, disabilities of the arm, kinesthetic sense, lymphedema, quality of life, shoulder and hand.

The number of women with breast cancer has been rapidly increasing and surgery, chemotherapy, and radiotherapy used in the treatment of breast cancer result in some problems, such as lymphedema (LE), which may affect the patient's quality of life..$^{[1]}$ The prevalence rates reported for LE that develop after breast cancer treatment vary between 0 and 56\%, depending on the diagnostic methods used. ${ }^{[2]}$

Although LE is not a life-threatening condition in most patients, it may cause various physical, psychological, and social problems. ${ }^{[3,4]}$ Swelling is the most prominent physical finding in the patients with LE. ${ }^{[5]}$ Edema may cause feeling of heaviness, tightness, and pain in these patients. Functional status of the affected upper limb and hand skills are important for the independence in daily life activities. In a recent study of LE in breast cancer patients, patients with extensive edema from arm to the hand had impaired kinesthetic sensation and hand function. ${ }^{[6]}$

Corresponding author: Hilal Yeşil, MD. Afyonkarahisar Sağlık Bilimleri Üniversitesi Tıp Fakültesi, Fiziksel Tip ve Rehabilitasyon Anabilim Dalı, 03030 Afyonkarahisar, Türkiye. e-mail: dradanur@yahoo.com 
There are several studies about kinesthetic sense, which is defined as the ability of sensing the movement of a joint (or an extremity), and many have shown that this sense is stimulated by the restriction of muscle spindle's ability to perceive movement primarily as a result of the action of muscle spindles with the contribution of receptors on the skin. ${ }^{[7]}$ In daily practice, the kinesthetic sense is measured as the smallest change required to elicit awareness of movement in the joint. Kinesthetic sense is particularly important for hand skills. ${ }^{[6]}$

Complex decongestive therapy (CDT) is the gold-standard method in the treatment of LE. ${ }^{[8]}$ Along with a significant volume reduction (50 to $70 \%$ ), CDT also reduces skin fibrosis and improves skin status, improves functional status, increases the quality of life by relieving the symptoms of the patient, and reduces the risk of developing cellulitis and angiosarcoma. ${ }^{[8,9]}$ Treatment includes skin care, manual lymphatic drainage (MLD), and compression therapy which are applied in two phases. ${ }^{[10]}$

Review of the literature reveals no study investigating the effects of CDT on the kinesthetic sense of hand in patients with BCRL, which is yet to be unveiled. In the present study, therefore, we aimed to investigate the effect of CDT on the kinesthetic sense of hands, upper extremity function, and the quality of life in patients with BCRL.

\section{PATIENTS AND METHODS}

This single-arm, pre-post intervention was conducted at Afyonkarahisar Health Sciences University and Ege University, Faculty of Medicine, Department of Physical Medicine and Rehabilitation between August 2018 and August 2019. A total of 50 women with BCRL (mean age: $56.5 \pm 9.6$ years; range, 36 to 71 years) who were admitted to our center for physical rehabilitation and received CDT due to BCRL (Stage 2-3) with edema in their hands and with dominant extremity involvement were included in this study. Those having recurrent cancer, acute deep venous thrombosis, severe cardiac and pulmonary problems, and open wound and infections in the affected extremity, and patients undergoing therapy for LE within the past six months or having cognitive deficits that would infer responding the questions were excluded from the study. The study flow chart is shown in Figure 1. A written informed consent was obtained from each patient. The study protocol was approved by the Ege University, Faculty of Medicine Ethics Committee (date/no: 16-5.1/15). The study was conducted in accordance with the principles of the Declaration of Helsinki. All patients underwent 20 sessions of CDT daily for $1 \mathrm{~h}$ over a total of four weeks. This program was composed of patient education, MLD, compression therapy with a short-stretch bandage for $23 \mathrm{~h}$ per day, exercise, and skin care. The MLD massage was performed daily for $30 \mathrm{~min}$, followed by multi-layered decongestive bandaging, and the patients were instructed to perform remedial exercises for LE, while the bandages were in place.

The treatment responses were evaluated at baseline and at four weeks after the initial examination. The demographic and clinical characteristics of the patients were recorded at the beginning of the treatment. The kinesthetic sense of the hand and hand grip strength in the affected arm, arm volume, functional status of the affected arm and health-related quality of life of the patients were evaluated before and after the treatment.

The kinesthetic sense was evaluated as the ability to copy different hand positions. The evaluations were performed using two different methods by providing visual and kinesthetic cues. The examined hand of the patient was positioned with the help of a masking plate to cover the patient's vision. While the patient's vision was covered, a total of nine assessments were performed, first one as a trial. In the visual control test, eight different hand positions were cued and the

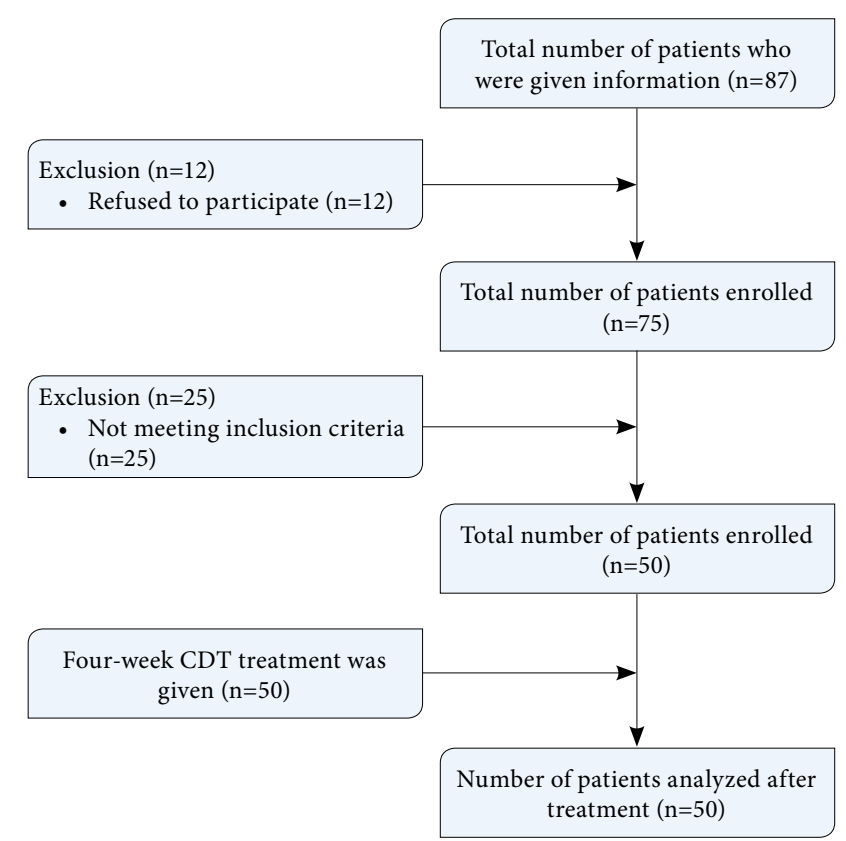

Figure 1. Flow diagram of the study.

CDT: Complex decongestive therapy. 
patient was asked to reproduce the hand figures. In the assessment of kinesthetic test, both examined and the other hand of the patient were positioned to cover the patient's vision. The untested hand was positioned by the physician. The patient was asked to copy position of the untested hand with the tested hand. Both affected and intact arms were evaluated. The result was scored on a scale from 0 to 3 points. ${ }^{[6]}$

A Jamar hand dynamometer was used to evaluate the hand grip strength. The measurement was performed, while the patient was in the seated position with the elbow placed on the table, shoulder adducted and in neutral rotation, elbow joint in $90^{\circ}$ flexion, and forearm and wrist in neutral position. The highest score in three trials was recorded. ${ }^{[1]}$

For the evaluation of edema, LE of the limbs were assessed by the same physiotherapist using the circumferential and volumetric methods before and after the treatment. Circumferential upper limb measurements were carried out with the arm abducted at $30^{\circ}$, starting at the level of the carpometacarpal joint, every $5 \mathrm{~cm}$ proximal to this point along both limbs. Afterwards, we used a computer program (limb volumes professional version 5.0) to convert these values into limb volumes in $\mathrm{mL} .{ }^{[12]}$

Functional status of the arm was evaluated from the perspective of the patient using the Disabilities of the Arm, Shoulder and Hand (DASH) score. The DASH was developed by the Institute of Work and Health and Ontario and the American Academy of Orthopaedic Surgeons to evaluate upper extremity problems. ${ }^{[13]}$ The questionnaire evaluates the functional status of the entire upper extremity with sports and musicians' module filled on a voluntary basis. It consists of 30 items related to the symptoms and daily life activities and takes around $5 \mathrm{~min}$ to complete, and it has a moderate scoring difficulty. All items are scored from 1 to 5 points ( $1=$ no difficulty, $5=$ unable to perform the activity). The total score ranges from 20 to 100 points, and higher scores indicate a greater level of disability.

The quality of life was evaluated using the European Organization for Research and Treatment of Cancer Quality of Life Core Questionnaire (EORTC QLQ-C30). This questionnaire was administered to evaluate the quality of life in patients with breast cancer, and consists of 30 questions and assesses symptoms which occurred in the previous two weeks. The items are rated on a Likert-type scale: 1- not at all, 2- a little, 3-quite a bit, 4-very much. The questionnaires are divided into three scales:
Global Health Scale (GHS), Functional Scale (FS), and Symptom Scale (SS) ${ }^{[14]}$

\section{Statistical analysis}

Statistical analysis was performed using the IBM SPSS version 22.0 (IBM Corp., Armonk, NY, USA) and MedCalc software (Medcalc Software, Belgium). Continuous variables were expressed in mean \pm standard deviation (SD) or median (min-max), while categorical variables were expressed in number and percentage. Comparisons of pre- versus post-intervention values for continuous variables were made using the paired t-test. The kinesthetic sense loss was examined using the McNemar's test. The relationships between continuous variables were analyzed using the Pearson and Spearman correlation analyses. A $p$ value of $<0.05$ was considered statistically significant.

\section{RESULTS}

Baseline demographic and clinical characteristics of the patients are shown in Table 1.

Changes in the volume of involved limbs and EORTC QLQ-C30 scores, kinesthetic sense scores of the hand, and DASH scores after treatment are presented in Table 2. The mean volumes of the limbs before and after treatment protocol were 3,920.1 $\pm 897.9 \mathrm{~mL}$ and 3,297.6 $\pm 773.1 \mathrm{~mL}$, respectively, indicating a statistically significant reduction in the volume of the involved limbs after the treatment $(\mathrm{p}<0.001)$.

There was a significant improvement in the GHS and FS scores of the EORTC QLQ-C30 ( $<<0.001$ and $\mathrm{p}=0.012$, respectively) and a significant reduction in the SS scores $(p<0.001)$. The mean DASH score decreased from $48.5 \pm 30.5$ at baseline to $39.9 \pm 21.9$ after treatment, showing an improvement in the DASH scores after the treatment $(\mathrm{p}=0.016)$.

In addition, the patients had significant visual and kinesthetic sense scores after the treatment $(\mathrm{p}=0.008$ and $\mathrm{p}<0.001$, respectively). Also, the kinesthetic sense loss rate of the patients significantly decreased after the treatment $(p=0.035$ and $p=0.001$, respectively) (Table 3). However, we found no significant correlation between the volume change and visual $(\mathrm{r}=-0.098 ; \mathrm{p}=0.499)$, and kinesthetic sense $(\mathrm{r}=-0.157 ; \mathrm{p}=0.277)$ change after the treatment.

None of the patients experienced adverse effects related to the treatment, and no additional drugs were used for LE during the study. 


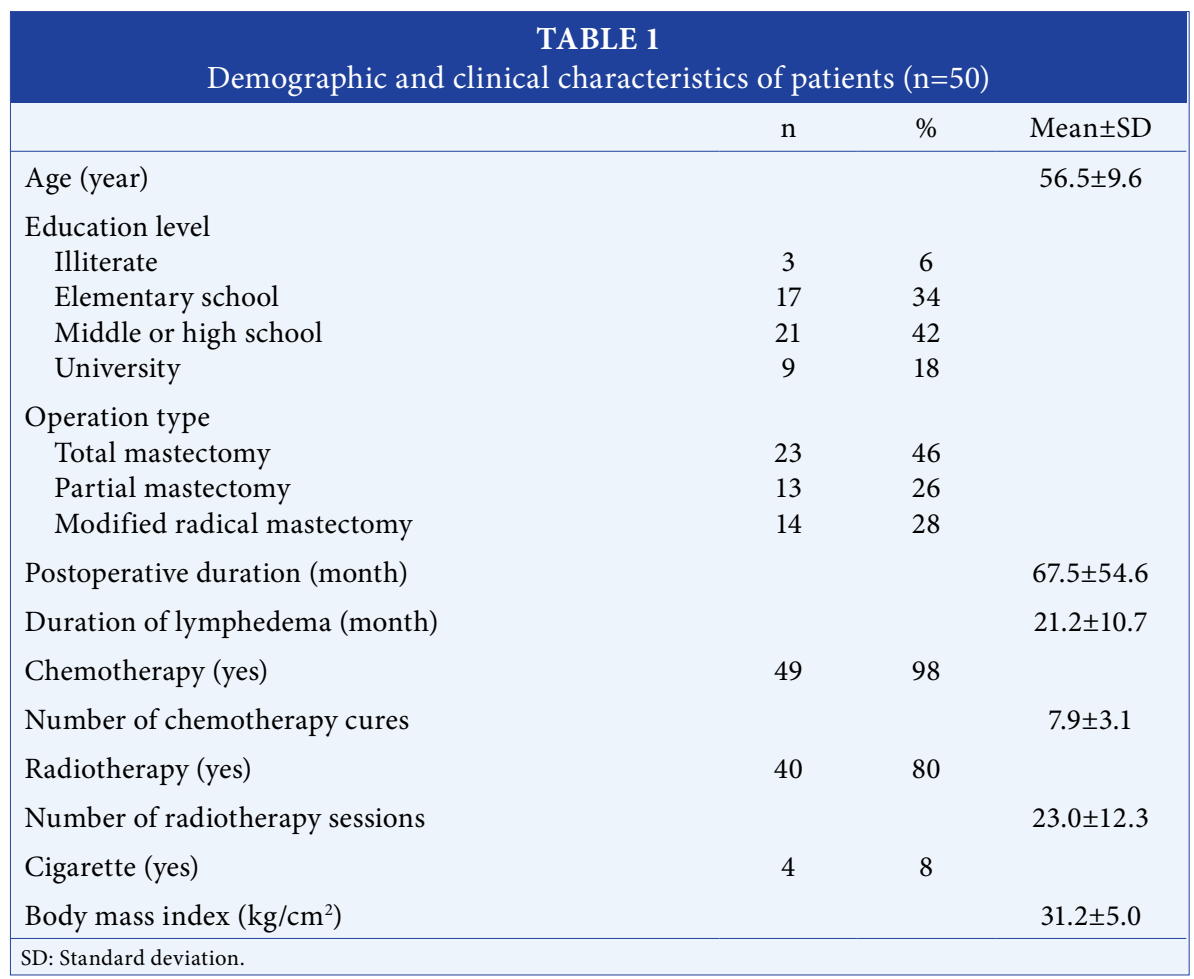

\begin{tabular}{|c|c|c|c|}
\hline \multicolumn{4}{|c|}{$\begin{array}{c}\text { TABLE } 2 \\
\text { Change in volume of involved limbs, and EORTC-QLQ-C30 and DASH scores after } \\
\text { treatment }\end{array}$} \\
\hline Variables & $\begin{array}{l}\text { Before treatment } \\
\text { Mean } \pm \text { SD }\end{array}$ & $\begin{array}{l}\text { After treatment } \\
\text { Mean } \pm \text { SD }\end{array}$ & $p$ \\
\hline Affected extremity volume (mL) & $3920.1 \pm 897.9$ & $3297.6 \pm 773.1$ & $<0.001$ \\
\hline EORTC-Q30-functional & $72.4 \pm 18.6$ & $77.4 \pm 13.7$ & 0.012 \\
\hline EORTC-Q30-symptom & $26.5 \pm 12.7$ & $20.9 \pm 11.3$ & $<0.001$ \\
\hline EORTC-Q30-global health & $58.0 \pm 18.3$ & $70.7 \pm 17.9$ & $<0.001$ \\
\hline DASH & $48.5 \pm 30.5$ & $39.9 \pm 21.9$ & 0.016 \\
\hline Jamar & $14.2 \pm 6.6$ & $14.1 \pm 4.7$ & 0.897 \\
\hline
\end{tabular}

\section{TABLE 3}

Change in kinesthetic sense score, and kinesthetic sense loss ratio after treatment

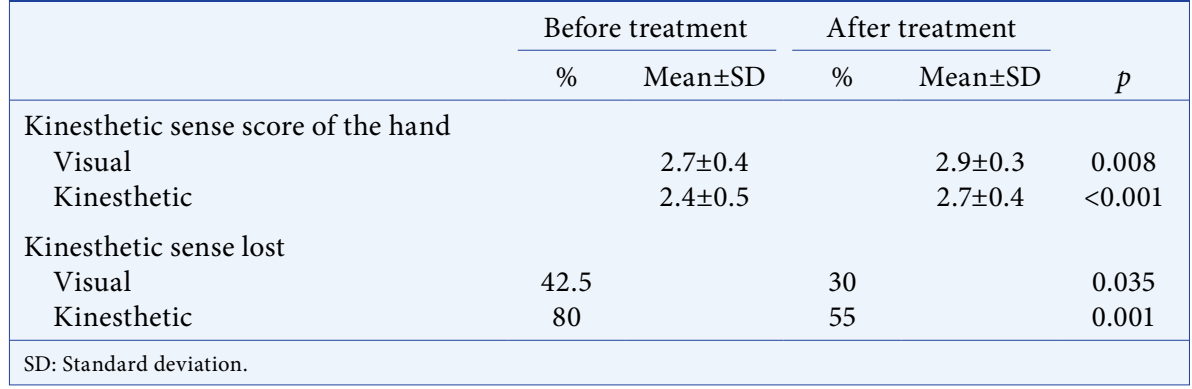




\section{DISCUSSION}

In this study, we evaluated the effect of CDT on the kinesthetic sense of hands, upper extremity function, and the quality of life in patients with BCRL. Our study results showed that CDT significantly improved both visual and the kinesthetic sense of the hands in patients receiving CDT treatment and CDT had significant effects on the arm volumes, functional status of the upper extremity, and the quality of life of the patients.

It has been well documented that LE affects many aspects of the human body, such as sensorial deficits, pain, loss of strength, restricted mobility, the tendency to infection, and skin tenderness in the affected limb. ${ }^{[15-17]}$ In the presence of edema in the hand accompanying the arm edema which occurs in 60 to $70 \%$ of patients with LE in the upper limb, a significant loss may occur in the hand functions. ${ }^{[6]}$ Consequently, this loss interferes with the patient's ability to perform daily life activities or necessitates external support and adversely affects the patient's quality of life. ${ }^{[18,19]}$ Currently, CDT is considered the gold standard in the treatment of LE. ${ }^{[8]}$ Several studies to date have investigated the effectiveness of CDT in treating and preventing the development of post-mastectomy LE. ${ }^{[20-22]}$ The study by Noh et al. ${ }^{[9]}$ evaluated the effectiveness of CDT in edema in 35 upper limbs of patients with LE and they found a significant improvement in the arm volumes. In another study, Yamamoto and Yamamoto ${ }^{[23]}$ reported a median reduction of $328.7 \mathrm{~mL}$ in the upper extremity volume following CDT with a rate of median volume reduction of $58.9 \%$. According to the results of a systematic review of 26 studies on CDT published from 2004 through 2011, CDT was found to reduce the extremity volume. ${ }^{[8]}$ Similar to these studies, in the present study, we found a significant improvement in the extremity volume with CDT in the patients with a mean LE duration of $21.2 \pm 10.7$ months.

Health-related quality of life has become more important with an increasing survival of patients with breast cancer thanks to the introduction of modern treatment options. ${ }^{[24,25]}$ Volume reduction achieved by CDT has resulted in an improvement in physical functions and quality of life. ${ }^{[8]}$ The study by Noh et al. ${ }^{[9]}$ evaluated 35 upper extremities of patients with LE and reported a significant improvement in the quality of life using Short Form-36 health survey. Similarly, prospective cohort studies conducted by Mondry et al. ${ }^{[26]}$ and Yesil et al. ${ }^{[12]}$ reported an increase in the quality of life score using CDT. According to the results of a recent review, exercise and CDT were associated with the most significant improvements in the health-related quality of life regarding targeted treatments for BCRL. ${ }^{[27]}$ Similar to those reported in previous studies, our findings showed a significant improvement in all scales of EORTC QLQ-C30 and consider that CDT was effective in improving the quality of life of the patients with LE.

Upper extremity functions decline as a result of joint motion limitation, weakness, paresthesia, rotator cuff tendonitis, and pain caused by upper extremity LE. ${ }^{[28]}$ Physical impairment in the upper extremities contributes to limitations in activities requiring the use of the affected upper limb. ${ }^{[29,30]}$ A limited number of studies has reported that CDT contribute to the improvement in upper extremity functions in patients with LE. ${ }^{[31]}$ Buragadda et al. ${ }^{[31]}$ suggested that CDT combined with the home-based program was more effective treatment than conventional therapies such as MLD, bandaging, glenohumeral joint mobilization, and respiratory exercises to reduce edema and improve upper extremity functions in patients with LE after mastectomy. In the present study, we showed a significant improvement with CDT in the extremity functions as assessed by DASH scores before and after the treatment.

The term kinesthesia refers to the perception of body parts in the space and recognizes the roles of the other parts of the body, and this term is used to express the conscious awareness of the body parts and their movements. ${ }^{[32]}$ In addition, kinesthesia plays a role in the hand skills and performance of movements. ${ }^{[33]}$ The impairment in the kinesthetic sense of the hand particularly affects hand functions. ${ }^{[34]}$ Hwang et al. ${ }^{[35]}$ compared patients with myelomeningocele aged between six and 12 years with healthy children regarding their kinesthetic sense and they reported that weakening in kinesthetic sense resulted in an impairment in hand function in children with myelomeningocele. Similarly, Minns et al. $^{[36]}$ demonstrated an inadequate kinesthetic sense as being one of the causes of insufficient hand functions in children with myelomeningocele.

Although many studies have investigated upper extremity function in patients with BCRL, only one study to date has evaluated the effects of edema on the kinesthetic sense of the hand. ${ }^{[6]}$ A cross-sectional study conducted by Karadibak and Yavuzsen ${ }^{[6]}$ assessed the functionality and the kinesthetic sense of the hand in women who developed BCRL. Kinesthetic sense of patients without edema in the hand was found to 
be better, compared to those with edema in the hand. The authors also observed loss of kinesthetic sense in $65.3 \%$ of patients who had coexisting impairment in performing daily life activities.

Considering that hand skills and performance are one of the important causes which affect the quality of life in patients with BCRL, it is reasonable to expect an increase in the performance with therapies provided to these patients. The present study, therefore, examined whether CDT used as the gold-standard method improved the kinesthetic sense of the hand in our LE patients with edema in their hands, and with dominant extremity involvement. Therefore, we used a test method which was comprised of eight different hand positions developed by Lynch et al. ${ }^{[33]}$ and adapted by Grant and Watter ${ }^{[37]}$ to evaluate the kinesthetic sense of the hand and we observed a significant improvement both in visual and kinesthetic functions after the treatment, compared to pretreatment values. However, we found no significant correlation between the volume change and kinesthetic sensation change after treatment. We attribute these results to various kinesthetic interventions such as massage and bandaging (i.e., short stretch produces a friction against the skin, as the patient moves) in CDT treatment, rather than volume decline in these patients. However, we found a significant negative correlation between the DASH scores before treatment and both visual and kinesthetic sense, suggesting that patients with impaired kinesthetic sense showed poorer upper extremity physical functions than patients with an intact kinesthetic sense. Based on these findings, we consider that rehabilitation programs focusing on improving upper extremity functions in daily life activities in patients with BCRL should also pay attention to kinesthetic sense.

Nonetheless, there are some limitations to this study. First, the relatively low number of patients in the study restricts the scope of the study. Second, we were only able to evaluate the short-term results of the patients. Further large-scale, longitudinal followup studies are warranted to confirm our findings and show the long-term effects of CDT. Although patient compliance and attendance rates were good, the hospital setting is another limitation. Additionally, hand edema was unable to be measured, although kinesthetic sense evaluation was performed and, probably, this resulted in the lack of a significant correlation between the decrease in the upper limb volume and kinesthetic sensory changes. Despite the limitations, on the other hand, we should note that this preliminary study is the first cohort study in the literature to evaluate the efficacy of CDT on kinesthetic sense in patients with BCRL.

In conclusion, BCRL is a serious complication which may lead to symptoms, such as pain, functional loss, weakness, fatigue, psychosocial problems, and eventually deterioration in the quality of life. In this study, significant improvements were observed concerning kinesthetic sense of hand, upper extremity functions, arm volume and the quality of life of patients with BCRL. These findings suggest that CDT is an effective and safe method to achieve not only a significant volume reduction in limbs involved by LE, but also favorable outcomes in the management of the health problems that are addressed in this paper.

\section{Declaration of conflicting interests}

The authors declared no conflicts of interest with respect to the authorship and/or publication of this article.

Funding

The authors received no financial support for the research and/or authorship of this article.

\section{REFERENCES}

1. Rogan S, Taeymans J, Luginbuehl H, Aebi M, Mahnig S, Gebruers N. Therapy modalities to reduce lymphoedema in female breast cancer patients: a systematic review and metaanalysis. Breast Cancer Res Treat 2016;159:1-14.

2. Das N, Baumgartner RN, Riley EC, Pinkston CM, Yang D, Baumgartner KB. Treatment-related risk factors for arm lymphedema among long-term breast cancer survivors. J Cancer Surviv 2015;9:422-30.

3. Gärtner R, Jensen MB, Kronborg L, Ewertz M, Kehlet H, Kroman N. Self-reported arm-lymphedema and functional impairment after breast cancer treatment--a nationwide study of prevalence and associated factors. Breast 2010;19:506-15.

4. Chachaj A, Małyszczak K, Pyszel K, Lukas J, Tarkowski R, Pudełko $\mathrm{M}$, et al. Physical and psychological impairments of women with upper limb lymphedema following breast cancer treatment. Psychooncology 2010;19:299-305.

5. Tiwari P, Coriddi M, Salani R, Povoski SP. Breast and gynecologic cancer-related extremity lymphedema: a review of diagnostic modalities and management options. World J Surg Oncol 2013;11:237.

6. Karadibak D, Yavuzsen T. Evaluation of kinesthetic sense and hand function in women with breast cancer-related lymphedema. J Phys Ther Sci 2015;27:1671-5.

7. Kito T, Hashimoto T, Yoneda T, Katamoto S, Naito E. Sensory processing during kinesthetic aftereffect following illusory hand movement elicited by tendon vibration. Brain Res 2006;1114:75-84.

8. Lasinski BB, McKillip Thrift K, Squire D, Austin MK, Smith $\mathrm{KM}$, Wanchai A, et al. A systematic review of the evidence for complete decongestive therapy in the treatment of lymphedema from 2004 to 2011. PM R 2012;4:580-601. 
9. Noh S, Hwang JH, Yoon TH, Chang HJ, Chu IH, Kim JH. Limb differences in the therapeutic effects of complex decongestive therapy on edema, quality of life, and satisfaction in lymphedema patients. Ann Rehabil Med 2015;39:347-59.

10. Vignes S, Porcher R, Champagne A, Dupuy A. Predictive factors of response to intensive decongestive physiotherapy in upper limb lymphedema after breast cancer treatment: a cohort study. Breast Cancer Res Treat 2006;98:1-6.

11. Schmidt RT, Toews JV. Grip strength as measured by the Jamar dynamometer. Arch Phys Med Rehabil 1970;51:321-7.

12. Yesil H, Eyigör S, Caramat İ, Işık R. Effects of complex decongestive therapy on quality of life, depression, neuropathic pain, and fatigue in women with breast cancer-related lymphedema. Turk J Phys Med Rehabil 2017;63:329-34.

13. Hudak PL, Amadio PC, Bombardier C. Development of an upper extremity outcome measure: the DASH (disabilities of the arm, shoulder and hand) [corrected]. The Upper Extremity Collaborative Group (UECG). Am J Ind Med 1996;29:602-8.

14. Aaronson NK, Ahmedzai S, Bergman B, Bullinger M, Cull A, Duez NJ, et al. The European Organization for Research and Treatment of Cancer QLQ-C30: a qualityof-life instrument for use in international clinical trials in oncology. J Natl Cancer Inst 1993;85:365-76.

15. Dominick SA, Natarajan L, Pierce JP, Madanat $H$, Madlensky L. The psychosocial impact of lymphedemarelated distress among breast cancer survivors in the WHEL Study. Psychooncology 2014;23:1049-56.

16. Shih YC, Xu Y, Cormier JN, Giordano S, Ridner SH, Buchholz TA, et al. Incidence, treatment costs, and complications of lymphedema after breast cancer among women of working age: a 2-year follow-up study. J Clin Oncol 2009;27:2007-14.

17. Ridner SH, Bonner CM, Deng J, Sinclair VG. Voices from the shadows: living with lymphedema. Cancer Nurs 2012;35:E18-26.

18. Caffo O, Amichetti M, Ferro A, Lucenti A, Valduga F, Galligioni E. Pain and quality of life after surgery for breast cancer. Breast Cancer Res Treat 2003;80:39-48.

19. Voogd AC, Ververs JM, Vingerhoets AJ, Roumen RM, Coebergh JW, Crommelin MA. Lymphoedema and reduced shoulder function as indicators of quality of life after axillary lymph node dissection for invasive breast cancer. Br J Surg 2003;90:76-81.

20. Smile TD, Tendulkar R, Schwarz G, Arthur D, Grobmyer $S$, Valente Set al. A review of treatment for breast cancerrelated lymphedema: paradigms for clinical practice. Am J Clin Oncol 2018;41:178-90.

21. Gradalski T, Ochalek K, Kurpiewska J. Complex decongestive lymphatic therapy with or without vodder II manual lymph drainage in more severe chronic postmastectomy upper limb lymphedema: A randomized noninferiority prospective study. J Pain Symptom Manage 2015;50:750-7.
22. Hwang WT, Chung SH, Lee JS. Complex decongestive physical therapy and low-level laser therapy for the treatment of pediatric congenital lymphedema: a case report. J Phys Ther Sci 2015;27:2021-2.

23. Yamamoto R, Yamamoto T. Effectiveness of the treatmentphase of two-phase complex decongestive physiotherapy for the treatment of extremity lymphedema. Int J Clin Oncol 2007;12:463-8.

24. Kaya T, Karatepe AG, Günaydn R, Yetiş H, Uslu A. Disability and health-related quality of life after breast cancer surgery: relation to impairments. South Med J 2010;103:37-41.

25. Rietman JS, Dijkstra PU, Debreczeni R, Geertzen JH, Robinson DP, De Vries J. Impairments, disabilities and health related quality of life after treatment for breast cancer: a follow-up study 2.7 years after surgery. Disabil Rehabil 2004;26:78-84.

26. Mondry TE, Riffenburgh RH, Johnstone PA. Prospective trial of complete decongestive therapy for upper extremity lymphedema after breast cancer therapy. Cancer J 2004;10:42-8.

27. Pusic AL, Cemal Y, Albornoz C, Klassen A, Cano S, Sulimanoff I, et al. Quality of life among breast cancer patients with lymphedema: a systematic review of patientreported outcome instruments and outcomes. J Cancer Surviv 2013;7:83-92.

28. Pinto M, Gimigliano F, Tatangelo F, Megna M, Izzo F, Gimigliano R, et al. Upper limb function and quality of life in breast cancer related lymphedema: a cross-sectional study. Eur J Phys Rehabil Med 2013;49:665-73.

29. Hayes SC, Janda M, Cornish B, Battistutta D, Newman B. Lymphedema after breast cancer: incidence, risk factors, and effect on upper body function. J Clin Oncol 2008;26:3536-42.

30. Hayes SC, Reul-Hirche H, Turner J. Exercise and secondary lymphedema: safety, potential benefits, and research issues. Med Sci Sports Exerc 2009;41:483-9.

31. Buragadda S, Alhusaini AA, Melam GR, Arora N. Effect of complete decongestive therapy and a home program for patients with post mastectomy lymphedema. J Phys Ther Sci 2015;27:2743-8.

32. McCloskey DI. Kinesthetic sensibility. Physiol Rev 1978;58:763-820.

33. Lynch MR, Raymer ME, Elvery JH, Walsh AL, Burns YR. The development of hand position sense. NZ Journal of Physiotherapy 1992;20:15-20.

34. Kara B, Yildirim Y, Karadýbak D, Acar U. Evaluation of the kinesthetic sense and function of the hand in early period in operated cervical disc hernia. Eur Spine J 2006;15:992-7.

35. Hwang R, Kentish M, Burns Y. Hand positioning sense in children with spina bifida myelomeningocele. Aust J Physiother 2002;48:17-22.

36. Minns RA, Sobkowiak CA, Skardoutsou A, Dick K, Elton RA, Brown JK. Upper limb function in spina bifida. Zeitschrift fur Kinderchirurgie 1977;22:493-506.

37. Grant L, Watter P. Ability to copy hand positions at 10 and 12 years of age. NZ Journal of Physiotherapy 1998;26:21-5. 